\title{
AJUSTE ESTRATÉGICO EN COOPERATIVAS DE AHORRO Y CRÉDITO COSTARRICENSES: UNA PROPUESTA
}

\author{
FEDERICO QUESADA CHAVES \\ Escuela de Ciencias de la Administración \\ Universidad Estatal a Distancia, Costa Rica \\ fequesada@uned.ac.cr
}

\section{RESUMEN}

Las cooperativas financieras en Costa Rica gozan de una tradición importante. El desempeño económico de éstas se traduce en un incremento de las condiciones de vida, tanto de aquellas personas que disfrutan de los beneficios de este tipo de organizaciones, como de los trabajadores que laboran para estas instituciones. En este documento se proponen los cimientos para una investigación cuyo objetivo es determinar el ajuste entre la estrategia seguida por este tipo de empresas y las prácticas de recursos humanos que éstas implementan. Se incorporan variables de recursos humanos y variables económicas que pueden afectar el desempeño económico de este tipo de empresas.

PALABRAS CLAVE: DESEMPEÑO FINANCIERO, RECURSOS HUMANOS, CAPACIDADES, ORGANIZACIÓN, COOPERATIVAS.

\section{ABSTRACT}

Costa Rican cooperatives have been part of an important national tradition thanks to their economic performance that have helped improve the living conditions of people who enjoy their benefits and of those working for those institutions. The adjustment of the strategies followed by these enterprises to the practices implemented in terms of human resources is determined. Both human resources as well as economic variable are incorporated in order to measure their impact on the economic performance of these enterprises.
KEYWORDS: FINANCIAL PERFORMANCE, HUMAN RESOURCES, CAPABILITIES, ORGANIZATION.

\section{INTRODUCCIÓN}

Este diseño de investigación propone establecer los parámetros para estudiar cómo se comportan las empresas cooperativas costarricenses de ahorro y crédito, en el diseño de sus prácticas de recursos humanos, y su posterior impacto sobre el rendimiento financiero de las mismas. Costa Rica ha disfrutado de una tradición cooperativa desde el siglo pasado y su impacto sobre el modo de vida costarricense ha sido considerado por varios actores, clave en la sociedad costarricense como positivo a lo largo de la historia. Con el pasar de los años y el avance de las tecnologías de la información y las comunicaciones, más empresas han optado por implementar best practices, las cuales, de ahora en adelante, serán entendidas como buenas prácticas de recursos humanos.

Existen varias teorías con respecto a la forma en que las buenas prácticas de recursos humanos, desde una perspectiva estratégica, se traducen en un mejoramiento del rendimiento financiero de la empresa. Entre ellos destaca el enfoque estratégico-contingente, el cual sostiene que las estrategias adoptadas por una empresa deberán condicionarse a la situación particular de la organización en un periodo determinado y de acuerdo con las bases culturales de ésta. 
Varias tendencias se han generado durante los últimos veinte años en torno al enfoque estratégico de recursos humanos y también se han hecho algunos intentos de cuantificar el proceso de ajuste con resultados dispersos. Schuller y Jackson (1987) han propuesto metodologías de análisis basadas en una tipología estratégica basadas en Porter (1985) utilizando la metodología de la casuística con resultados concluyentes a favor de este enfoque de ajuste.

Otros autores como Huselid (1995) han intentado demostrar igualmente el proceso de ajuste analizando muestras grandes y no han llegado a las mismas conclusiones. Cano y Céspedes (2003) intentan realizar el mismo análisis de ajuste estudiando muestras de empresas cooperativas. Estos últimos autores sostienen que es pertinente analizar el ajuste en empresas con estas características debido a que no operan con los mismos criterios que las empresas analizadas por sus contrapartes anglosajones y que, por lo tanto, resulta relevante estudiar sus implicaciones para este tipo de organizaciones debido a su naturaleza particular de funcionamiento.

Pfeffer y Veiga (1999) sostienen que todas aquellas empresas que visualizan a sus trabajadores como activos y les proporcionan capacitación, se encuentran con la posibilidad de alcanzar mayor éxito financiero que sus contrapartes que no aplican estas medidas, esto porque el ambiente propicio para el desarrollo de capital humano tiene un fuerte impacto sobre el desarrollo de ventajas competitivas y el mejoramiento de una situación financiera. Esta propuesta es lo que igualmente se entenderá como buenas prácticas de recursos humanos afines al funcionamiento de las empresas cooperativas.

Este artículo tiene como objetivo sentar las bases de un diseño de investigación cuyo propósito es analizar el impacto que tienen las buenas prácticas de recursos humanos y su impacto financiero en las empresas cooperativas costarricenses de aho- rro y crédito. Para ello, este documento se divide en cuatro partes: introducción, una sección dedicada a analizar la relevancia de este estudio sobre todo en países en vías de desarrollo, un marco teórico donde se analiza las principales corrientes teóricas que estudian este tema y finalmente, una sección dedicada a estudiar la metodología para realizar esta indagación así como algunos datos ilustrativos relacionados con el sector en Costa Rica.

\section{Relevancia}

La mayoría de los estudios realizados sobre dirección estratégica de recursos humanos han dedicado sus esfuerzos a dilucidar los problemas propios de organizaciones que operan en mercados altamente competitivos, esto se ha debido a la influencia anglosajona y el tipo de mercado en el que operan la mayoría de las organizaciones estudiadas, donde prevalece el carácter individual con fines de lucro por encima del bienestar social colectivo. La búsqueda de espacios comunes, en los cuales la teoría de dirección estratégica de recursos humanos pueda enriquecer el análisis de empresas con funcionamiento colectivo y más adaptadas a las condiciones presentes en países en vías de desarrollo, en los cuales imperan imperfecciones en uno o varios mercados simultáneamente, se vuelve una necesidad para el desarrollo económico de estos países. Un análisis que no se ha planteado y que valdría la pena considerar específicamente para Costa Rica viene delimitado en la siguiente pregunta:

¿Las empresas cooperativas de ahorro y crédito de Costa Rica que aplican buenas prácticas de recursos humanos logran mejor desempeño financiero en el tiempo que aquellas que no aplican estas medidas?

\section{MARCO TEÓRICO}

La literatura que atañe el tema de la estrategia de recursos humanos y su impacto en el desempeño es vasta y cuenta con una amplia variedad de 
definiciones y tendencias. Wright y Macmahan (1992) reconocen la necesidad de brindar articulación a un marco teórico muy disperso en una ciencia relativamente joven como lo es la ciencia de la dirección de empresas y, por lo tanto, brindan una definición de estrategia de recursos humanos como "el patrón de esfuerzos y actividades que realizan los recursos humanos planificados para permitir a una empresa alcanzar sus objetivos" (p. 297). Los autores proponen que la definición anterior tiene validez a nivel vertical porque permite un encadenamiento entre las prácticas de gerencia del recurso humano y el proceso organizativo. Adicionalmente, tiene relevancia a nivel horizontal porque permite establecer congruencia y coordinación entre las diferentes prácticas de recursos humanos. A este determinante en la estrategia, se le conoce como ajuste, el cual ha recibido un tratamiento distinto en diferentes investigaciones. Las diversas metodologías aplicadas han provocado múltiples resultados como lo demuestran Cano y Céspedes (2003) quienes afirman que "en general, los trabajos empíricos llevados a cabo proporcionan evidencias contrarias. No obstante, existen ciertos efectos positivos sobre el rendimiento cuando se produce el ajuste" (p. 65). El debate se centra en si realmente existe algún tipo de conexión entre las prácticas de recursos humanos y su impacto en la rentabilidad de la compañía el cual deviene de la aplicación de una estrategia específica.

El aumento en el rendimiento financiero puede verse incrementado por la aplicación de buenas prácticas de recursos humanos como plantean Pfeffer y Veiga (1999) y Huselid (1995). Estos autores también sugieren, que con la implementación de un ajuste, es decir un ligamen entre la estrategia de recursos humanos y la estrategia general de la empresa, el rendimiento financiero debería incrementarse aún más ceteris-paribus. El modelo estratégico contingente plantea que si se aplica una estrategia de recursos humanos que sea contingente con la estrategia de la em- presa, esto se traducirá en un aumento en el rendimiento financiero, sin embargo esta relación de interacción, es particular para cada empresa, debido a sus condiciones organizativas históricas, el entorno en el que se desenvuelve y las condiciones particulares de sus activos.

Schuller y Jackson (1987) proponen una vertiente del enfoque estratégico la cual estudia el encadenamiento entre las ventajas competitivas que tienen una organización y su coherencia en la aplicación en el desarrollo organizacional. Estos autores adicionalmente afirman que aquellas empresas que sean las primeras en introducir algún tipo de ventaja innovadora permanecerán en el mercado más tiempo y serán quienes dicten las direcciones que el resto del mercado seguirá. Las tres formas en las cuales puede ser introducida una estrategia a través de los recursos humanos es mediante la innovación (ofrecer un producto nuevo y diferente), incremento en la calidad y reducción de costos.

Las estrategias seleccionadas y el mercado en el cual se está interactuando serán los determinantes a la hora de realizar el diseño organizacional y de perfiles. Los autores afirman que los trabajadores tienen ciertos conocimientos y destrezas determinadas, así como también cuentan con conocimientos generales y habilidades comprobadas, ambos tipos de capacidades tendrán un impacto específico en el desarrollo de ventajas competitivas. También distinguen conductas específicas que deben tener los trabajadores que se desenvuelven en un ámbito social, Huselid (1995) afirma que las interacciones que se lleven a cabo entre los trabajadores y la aceptación serán factores determinantes a la hora de que se implementa el proceso de ajuste. Así por ejemplo, en el siguiente esquema se distinguen las siguientes estrategias y los diferentes roles identificados necesarios para poder implementarla según Schuller y Jackson (1987). 
TABLA 1

\section{ESTRATEGIAS Y CONDUCTAS POR PARTE DE LOS TRABAJADORES}

\section{Estrategia implementada}

Conductas requeridas por parte de los trabajadores

Estrategia de Innovación

Hipótesis propuesta:

Una empresa que persigue estrategias de innovación tendrá las siguientes características:

1. Puestos de trabajo que requieren interacción y coordinación entre

1. Alto grado de comportamiento creativo grupos de trabajo

2. Incentivos que reflejan alcances de un grupo en el largo plazo

3. Puestos de trabajo que favorezcan el desarrollo de destrezas que puedan ser utilizados en otras posiciones en la empresa

4. Sistemas de compensación que reflejan el desarrollo de interno más que el desarrollo externo

5. Pago de salarios es bajo pero permite a los empleados participar de diferentes tipos de incentivos como acciones de la empresa, pluses y opciones sobre acciones para compensar el salario bajo.

2. Enfocado en el largo plazo

3. Un relativamente alto comportamiento cooperativo e interdependiente

4. Preocupación moderada por la calidad

5. Preocupación moderada por la cantidad

6. Igualmente preocupado por procesos y resultados

7. Proclive a tomar riesgos

8. Alta tolerancia a lo impredecible y ambiguo

6. Amplios espectros de carrera que permiten el desarrollo de diferentes tipos de carreras y especializaciones

Estrategia de Mejoramiento en la calidad

Hipótesis propuesta:

1. Puestos de trabajo fijos y bien definidos

2. Altos niveles de participación por parte de los empleados en el proceso de toma de decisiones relacionadas con su puesto de trabajo

3. Retribuciones de corto plazo y orientadas a resultados tanto individuales como grupales

4. Trato igualitario entre empleados y estabilidad laboral

5. Capacitación y desarrollo extensiva de empleados
1. Conductas repetitivas y predecibles

2. Enfocado hacia el mediano y largo plazo

3. Moderadamente reducida conducta hacia el largo plazo

4. Preocupación moderada hacia la producción y la cantidad

5. Alta preocupación hacia la calidad

6. Alta preocupación por el proceso

7. Averso al riesgo

8. Comprometido con los roles de la compañía 
Estrategia de Reducción de Costos:

Hipótesis propuesta:

1. Puestos de trabajo fijos y estables con descripciones de trabajo específicas

2. Puestos de trabajo específicos e incentivos de superación que implican especialización y eficiencia

3. Retribuciones orientadas hacia el corto plazo y los resultados

4. Monitoreo del mercado de salario para realizar compensaciones

5. Niveles de capacitación y desarrollo mínimas.
1. Comportamiento repetitivo

2. Enfocado hacia el corto plazo

3. Actitud autónoma e individual

4. Preocupación moderada por la calidad

5. Alta preocupación por cantidad y producción

6. Enfocado hacia resultados

7. Averso al riesgo

8. Alto grado de satisfacción con la estabilidad

Fuente: Schuller y Jackson (1987).

El modelo de Schuller y Jackson (1987) constató sus hipótesis mediante una metodología basada en la casuística, y afirman que la selección de la estrategia más adecuada debe basarse en las características del recurso humano y del entorno en el que se desenvuelve. Huselid (1995) determina que existe relación entre el proceso de selección de prácticas en el trabajo, sin embargo sus resultados no son concluyentes con los de Schuller y Jackson (1987) ya que en sus hipótesis logra demostrar que existe algún tipo de relación entre los sistemas de buenas prácticas de recursos humanos, pero no logra demostrar que estos se encuentren alineados con una estrategia en particular, a pesar de que una de sus hipótesis fue planteada con ese fin. Huselid (1995) utilizó una muestra de casi mil empresas en los Estados Unidos, y un tratamiento de los datos mucho más robusto, mediante técnicas de regresión logística.

Huselid (1995) debate que Schuller y Jackson (1992) no integran en el análisis, la situación cualitativa del diseño organizacional, esto quiere decir que si un puesto de trabajo está diseñado con límites inflexibles, esta situación no permite modificar los procesos y procedimientos de manera que se desarrolle la ventaja competitiva. Por otro lado, este autor también sostiene que generalmente los trabajadores no encuentran incentivos a lo interno de la empresa para trabajar al máximo de sus capacidades individuales. El vínculo hallado por Huselid sostiene que si una empresa desarrolla una ventaja competitiva en el largo plazo, ésta debería traducirse en un incremento de la productividad de los trabajadores y, finalmente, esto debería desembocar en un mejoramiento de la situación financiera de la empresa', la cual debería mantenerse en el largo plazo. Este autor se preocupa especialmente por la rotación de trabajadores y su impacto en la productividad, afirmando que existe una relación inversa entre ambas variables, y destaca la existencia de una gran cantidad de determinantes que impactan la rotación de trabajadores tales como la edad, estabilidad percibida en el lugar de trabajo y presencia de un sindicato, entre muchas otras.

1. Esta teoría es válida sin embargo si se conjuga con situaciones favorables de entorno, por lo tanto se asume que la estrategia implementada por la empresa en ese momento es de un ambiente de negocios estable, o en el pico de un ciclo de negocios. 
Al existir tantas variables que explican el comportamiento de la rotación de los trabajadores, en este trabajo se presentaron dos problemas a la hora de implementar metodologías, uno de ellos fue que se debió considerar el tamaño de las industrias y su evolución, el otro, es que se debió utilizar una metodología que incorporara un análisis que incluyera todas las variables de manera conjunta. Otro punto destacado en el trabajo desarrollado por Huselid, el cual guía su segunda hipótesis de trabajo, es que deben existir sinergias y complementariedades entre las medidas para incrementar el desempeño y su interiorización por parte de los empleados. Esta situación los trabajadores la pueden percibir como margen de credibilidad de que si incrementan su desempeño, esto se traducirá en un mejoramiento de su situación laboral.

Las conclusiones alcanzadas en este estudio derivaron de las siguientes hipótesis:
Cano y Céspedes (2003) identifican el proceso de ajuste dentro de las cooperativas de ahorro y crédito, el cual se manifiesta de manera expresa mediante la coherencia existente entre una práctica de recursos humanos y su asociación con la estrategia de la empresa. Huselid (1995) identifica dos tipos de ajuste, uno interno y otro externo. El primero se refiere a las políticas adoptadas por una empresa y su consistencia a lo interno de ésta, la cual se refleja en el mejoramiento del desempeño. El segundo se refiere a la adopción de cualquier medida y estrategia en particular, ya que estos serán los determinantes de si esa medida ha sido apropiada o no. Cano y Céspedes (2003) utilizan el sistema consistente o de best practices debido a que Huselid; Schullery Jackson afirman que la evidencia empírica parece inclinarse en esta dirección.

Cano y Céspedes (2003) intentan determinar si existe efectivamente este ajuste en las empresas

TABLA 2

\section{HIPÓTESIS, METODOLOGÍA Y CONCLUSIONES}

\begin{tabular}{|c|c|c|c|}
\hline & Hipótesis & Metodología & Conclusiones \\
\hline $1 . B$ & $\begin{array}{l}\text { Los sistemas de alto desempeño en la práctica del trabajo dismi- } \\
\text { nuyen la rotación de trabajadores, incrementan la productividad y, } \\
\text { por lo tanto, mejoran la posición financiera de la empresa } \\
\text { La rotación de trabajadores y la productividad son variables que } \\
\text { median entre los sistemas de alto desempeño en la práctica del } \\
\text { trabajo y el mejoramiento de la situación financiera }\end{array}$ & \multirow{3}{*}{$\begin{array}{l}\text { Se tomó una muestra de } 968 \\
\text { empresas y se les aplicó un } \\
\text { cuestionario para determinar } \\
\text { cuáles eran las políticas de } \\
\text { recursos humanos aplicadas y } \\
\text { su coherencia con la estrate- } \\
\text { gia de la empresa. } \\
\text { A las variables detectadas } \\
\text { se le aplicaron modelos de } \\
\text { regresión lineal para deter- } \\
\text { minar su influencia sobre el } \\
\text { rendimiento. }\end{array}$} & $\begin{array}{l}\text { Se acepta que las prácticas de recursos } \\
\text { humanos mejoran la situación financiera de } \\
\text { la empresa }\end{array}$ \\
\hline 2. & $\begin{array}{l}\text { Las complementariedades y las sinergias entre las medidas de alto } \\
\text { desempeño en la práctica del trabajo disminuirán la rotación de } \\
\text { personal, incrementarán la productividad y el desempeño finan- } \\
\text { ciero. }\end{array}$ & & $\begin{array}{l}\text { Se comprueba que las sinergias resultan el } \\
\text { componente mediador entre las políticas de } \\
\text { recursos humanos y sus efectos en la situa- } \\
\text { ción financiera }\end{array}$ \\
\hline 3. & $\begin{array}{l}\text { El alineamiento entre los sistemas de alto desempeño en la } \\
\text { práctica del trabajo con la estrategia de la empresa disminuyen la } \\
\text { rotación de trabajadores, incrementan la productividad y mejoran } \\
\text { la posición financiera. }\end{array}$ & & $\begin{array}{l}\text { Los resultados obtenidos no son concluyen- } \\
\text { tes con respecto al ajuste interno y externo } \\
\text { entre las políticas de recursos humanos y su } \\
\text { alineamiento con la estrategia }\end{array}$ \\
\hline
\end{tabular}

Fuente: Cano y Céspedes (2003). 
cooperativas andaluzas, llegando a conclusiones distintas a las Huselid (1995). Estos autores logran reafirmar que existe algún tipo de ajuste entre las cooperativas que aplican prácticas de recursos humanos y estrategia de manera conjunta logran un mejor desempeño financiero. Sin embargo es importante rescatar que la muestra utilizada no es tan grande como la Huselid (1995) y a la hora de realizar su modelo de regresión, a pesar de que obtiene los signos esperados en los coeficientes, estos no son estadísticamente significativos, a lo cual los autores aducen que es necesario realizar estratificaciones de las cooperativas de acuerdo a su actividad y estudiar las variables externas que puedan afectar el entorno en el cual se desenvuelven este tipo de empresas según industria.

Según Cano y Céspedes (2003), sería necesario aislar una serie de variables externas relacionadas con la inversión-financiamiento, comercialización, producción y otras variables ligadas con el entorno, que al parecer este estudio y otros no consideran, y que tal vez no permiten aislar con certeza el impacto del ajuste entre los recursos humanos y el desempeño financiero. Otro punto que estos autores resaltan, y que resulta crucial para enriquecer el análisis del ajuste, es la presencia de un efecto retardado o rezagado en el tiempo, el cual puede ser evaluado mediante efectos rezagados en variables de series de tiempo lo cual obligaría a trabajar con datos de panel$^{2}$. Esto plantea un reto importante porque es muy difícil tener acceso a información histórica por parte de las empresas.

El modelo para esta investigación tiene el propósito de analizar el enfoque estratégico-contingente de recursos utilizando la tipología de estrategia de Schuller y Jackson (1992), con

2. Será conveniente realizar un análisis histórico así como también una recopilación de datos de corte transversal para poder construir una base de datos de panel que permita evaluar efectos rezagados. un matiz para analizar tanto variables que se encuentran en el entorno y que tienen una influencia en el comportamiento de instituciones financieras, tales como las tasas de interés activas y pasivas, el tipo de cambio y la cantidad de activos con los que cuenta la cooperativa para realizar sus operaciones. Por otro lado, para medir las tendencias de una política de recursos humanos alineada con una estrategia definida de una empresa y poder analizar si esta tiene un impacto en el mediano plazo, será necesaria la aplicación de técnicas de análisis dinámicas. A continuación se expone el modelo por analizar:

Como se puede observar en el esquema anterior, las empresas cooperativas costarricenses, que aplican sistemas de buenas prácticas de recursos humanos mejoran su situación financiera. El efecto rezago se incorpora en el modelo, debido a que la mayoría de las investigaciones en recursos humanos no consideran la variable tiempo, y es muy probable que medidas tomadas en un periodo determinado de tiempo, den resultados en periodos posteriores.

\section{CONCLUSIONES}

El proceso de ajuste entre la estrategia que siguen las organizaciones cooperativas de ahorro y crédito y su estrategia y política de recursos humanos, no ha sido investigada a profundidad en Costa Rica. Es necesario delimitar este comportamiento, debido al tamaño con el que cuenta este sector financiero en la economía costarricense y el potencial impacto que puede tener un incremento en la eficiencia de este tipo de instituciones. Por un lado, el mejoramiento del desempeño financiero se puede traducir en un mayor beneficio para todas aquellas personas que confían en las cooperativas de ahorro y crédito como alternativa a otros servicios financieros. Adicionalmente, con la aplicación de este tipo de medidas a lo interno de la empresa, se 


\section{AJUSTE INTERNO Y VARIABLES EXTERNAS QUE AFECTAN EL ENTORNO DE LAS COOPERATIVAS DE AHORRO Y CRÉDITO}

AJUSTE INTERNO*

\begin{tabular}{|l|}
\hline ESTRATEGIA DE INNOVACIÓN \\
Variables consideradas $\left(1 \ldots n_{\mathrm{t}}\right)$ \\
Variables consideradas $\left(1 \ldots \mathrm{n}_{\mathrm{t}-1}\right)$ \\
Variables consideradas $\left(1 \ldots . \mathrm{n}_{\mathrm{t}-2}\right)$
\end{tabular}

ESTRATEGIA DE CALIDAD

Variables consideradas (1...n $)$ Variables consideradas $\left(1 \ldots \mathrm{n}_{\mathrm{t}-1}\right)$ Variables consideradas $\left(1 \ldots \mathrm{n}_{\mathrm{t}-2}\right)$

Variables consideradas $\left(1 \ldots n_{\mathrm{t}}\right)$

Variables consideradas $\left(1 \ldots n_{t-1}\right)$

Variables consideradas $\left(1 \ldots . \mathrm{n}_{\mathrm{t}-2}\right)$

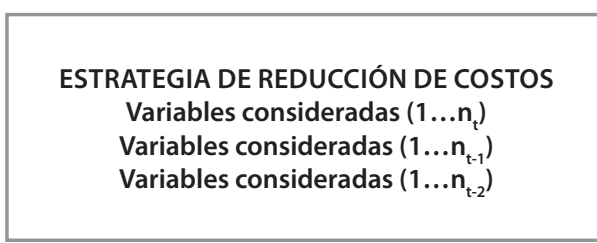

Cantidad de activos de la organización al período $t$. (variable de control)
VARIABLES EXTERNAS

Tasa de interés básica pasiva en el período actual (comercialización)

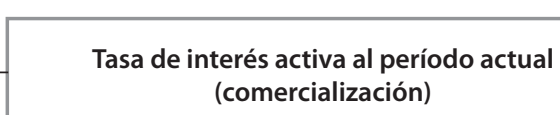
(comercialización)

trats

Tipo de cambio al período actual
(riesgo)

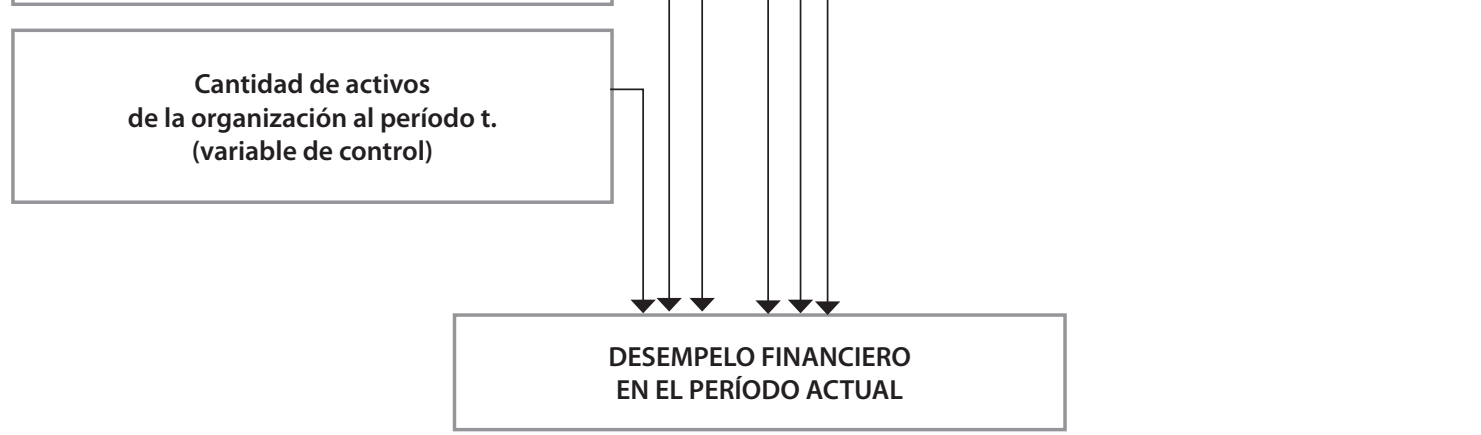

* Las variables consideradas son aquellas denotadas en el cuadro uno y propuestas por Schuller y Jackson. 
podría esperar mayor innovación e incremento en las capacidades organizativas.

Existen una gran cantidad de investigaciones pendientes en el sector cooperativo costarricense, la propuesta de este documento, es tan solo una de tantas indagaciones que es necesario realizar en todas las cooperativas de Costa Rica, para aproximar incrementos en la competitividad de este tipo de empresas, entendido este concepto dentro del ámbito en el cual se mueven las cooperativas, el cual es muy distinto al contexto en el cual se mueven las pequeñas, medianas y grandes empresas, cuyo fin es el lucro. El desarrollo de este tipo de indagaciones, es necesario para la construcción de nueva teoría relacionada con el sector cooperativo.

También destaca la necesidad de realizar investigaciones en el sector cooperativo que trasciendan el enfoque de corte transversal. Estudios mediante la recopilación de datos de panel y la observación de la evolución en el tiempo de diferentes instituciones, puede proporcionar una mayor cantidad de datos a lo largo del tiempo, que la observación en un momento determinado. Estudios de corte transversal son la norma no solo en este tipo de investigaciones, sino también en la mayoría de las líneas desarrolladas alrededor de la ciencia de la dirección de empresas. El desarrollo de series de tiempo relacionadas con variables organizativas y de políticas empresariales es necesario para proporcionar mayor robustez a las teorías vigentes en estos temas.

\section{Futuras líneas de investigación}

El desarrollo de una agenda de investigación que permita delimitar la construcción de nueva teoría en relación a este tema, debe considerar las condiciones particulares de este sector de la economía. Los estudios previos, se han preocupado por investigar distintas áreas de los recursos humanos para determinar las estrategias de recursos humanos, sin embargo la estrategia seguida por las organizaciones investigadas se encuentra subordinada al tipo de objetivo que persiguen este tipo de empresas, el cual es el lucro. Para adaptar esta investigación a objetivos cooperativos, es necesario primero realizar una investigación sobre los objetivos que siguen cada una de este tipo de empresas y lograr una armonización de objetivos.

Una vez determinados cuáles son los objetivos de este tipo de empresas, sería necesario realizar la investigación propuesta en este documento y en un tercer momento, sería necesario investigar los objetivos de todas las cooperativas de Costa Rica con datos de panel, clasificándolas por Industrias, para posteriormente, determinar si existe un ajuste entre la estrategia de todas las cooperativas de Costa Rica y sus políticas y estrategias de recursos humanos.

Para la validación de este modelo, en una futura investigación, es necesaria una comprobación empírica de tipo cuantitativa. Para este tipo de investigación, resulta necesario el planteamiento de un cuestionario que permita consultar a las empresas cooperativas directamente (fuente primaria) las variables internas que determinan el comportamiento de sus recursos humanos según la estrategia seleccionada, ya que en estos momentos no existen indicadores relacionados con el tema que se desea investigar. También será necesario recopilar información sobre las variables que pueden afectar el entorno de este tipo de empresas en Costa Rica, dado que a pesar de que la mayor cantidad de actividades son realizadas en el mercado local, se encuentran expuestas a variables del entorno financiero nacional e internacional y sus fluctuaciones.

Para analizar las estrategias implementadas, y su posterior clasificación, se sugiere utilizar una metodología de análisis de conglomerados debido a que Cano y Céspedes (2003) utilizan esta metodología para clasificar estrategias de acuer- 
do con un tipología de estrategia basada en Miles y Snow. Sin embargo, si se considera el uso de modelos de regresión dinámicos resulta más pertinente utilizar la tipología de Schuller y Jackson (1987), debido a que es más probable que este tipo de estrategias y el correspondiente diseño organizacional, especificación de perfiles e implementación tengan un impacto retardado o rezagado, suponiendo que existe poca rotación de trabajadores.

Finalmente, para analizar la influencia de las prácticas de recursos humanos, su alineamiento con la estrategia y el impacto sobre el desempeño financiero, se recomienda el uso de modelos de regresión dinámicos rezagados a uno y dos periodos considerando no solo las estrategias aplicadas, sino también variables externas, como tipo de cambio, tasas de interés activas y pasivas y el número de activos presentes en las cooperativas.

\section{REFERENCIAS}

Cano, C. J. \& Céspedes, J.J. (2003). Estrategia de Negocios y Prácticas de Recursos Humanos en las Empresas Cooperativas. Revista de Economía Pública, Social y Cooperativa, 46, 63-94.

Huselid, M.A. (1995). The impact to human resources management practices on turnover, productivity, and corportate financial performance. Academy of Management Journal, 38( 3), 635-665.

Porter, M. (1985). Competitive Advantage. New York: Free Press.

Pfeffer, J. \& Veiga, J.F. (1999). Putting people first for organizational success. Academy of Management Executive, 13(2), 37-48.

Schuller, R. \& Jackson, S. (1987). Linking competitive strategies with human resources management practices The Academy of Management Executive, 1( 3), 207-219.

Wright, P.M. \& Mcmahan, G.C. (1992). Theoretical Perspectives for Estrategic Human Resources Management. The Journal of Management, 18(2), 295-320.

Recibido: 21-06-2010

Aceptado: 12-09-2010 